\title{
Autophagic membrane delivery through ATG9
}

\author{
Cell Research (2017) 27:161-162. doi:10.1038/cr.2017.4; published online 10 January 2017
}

One of the key questions regarding macroautophagy/autophagy is the mechanism through which the transmembrane protein ATG9 functions in delivering membrane to the expanding phagophore, the sequestering compartment that matures into an autophagosome. In a recent study, Zhou et al. identified a novel mechanism that regulates ATG9 trafficking from the plasma membrane and trans-Golgi network, which involves two conserved sorting signals required for ATG9 interaction with the AP1/2 adaptor complex and phosphorylation of ATG9 at Tyr8 by SRC kinase and at Ser14 by ULK1 for proper function during basal and starvation-induced autophagy.

Autophagy is a self-eating process in which cells engulf portions of the cytosol or organelles in phagophores. After phagophore maturation, the resulting double-membrane autophagosomes deliver the cargo to the lysosome or vacuole, where it is ultimately degraded; the resulting breakdown products are released back into the cytosol and recycled to allow the cells to survive unfavorable conditions and maintain cellular homeostasis. The autophagy process can be divided into several steps involving different autophagy-related (ATG) proteins. The ULK1/2 kinase complex including ULK1/2, ATG13, RB1CC1/FIP200 and ATG101 is activated to induce autophagy, whereas the ATG9 system is required for phagophore expansion [1].

Previous studies have indicated multiple membrane sources in contributing to autophagosome formation, including the endoplasmic reticulum, the Golgi apparatus and the plasma membrane [2-7]. ATG9 is the only transmembrane protein in the autophagy core machinery and has been proposed to play a key role in directing membrane from donor organelles for autophagosome formation. Thus, understanding the mechanisms that regulate the movement of ATG9 is fundamental in order to gain a mechanistic insight into autophagy.

Previous studies have identified phosphorylation as an important posttranslational modification that controls Atg9 trafficking in yeast $[8,9]$. The work by Zhou et al. [10] reveals a novel mechanism of mammalian ATG9 trafficking regulation in basal and induced autophagy that involves both adaptor protein sorting signals and phosphorylation. Putative tyrosine- and dileucinebased sorting signals, $\mathrm{Y}_{8} \mathrm{QRL}_{11}(\mathrm{SS} 1)$ and $\mathrm{EEDL}_{25} \mathrm{~L}_{26}(\mathrm{SS} 2)$, were identified in the N-terminus of ATG9; mutations of either or both to alanine weakened or totally abolished the interaction of ATG9 with the AP1/2 complex, based on in vivo and in vitro affinity studies. These mutations also affected the juxtanuclear distribution of ATG9 and its localization at the trans-Golgi network (TGN). A cell surface biotinylation assay and immunogold-labeled EM analysis showed that ATG9 sorting signal mutants were retained at the plasma membrane due to a severe endocytosis defect.

Zhou et al. predicted that recognition of these sorting signals might be regulated by phosphorylation, and they determined that Tyr8 of ATG9 is directly phosphorylated by SRC kinase. This phosphorylation event promotes the interaction of ATG9 with the AP1/2 complex and leads to the movement of ATG9 away from the juxta-nuclear region. SRC can be activated in response to stimulation by the EGFR (epidermal growth factor receptor), thus leading to increased ATG9 phosphorylation at Tyr8 and enhanced interaction with the AP2 complex, suggesting that ATG9 is internalized from the plasma membrane through an endocytic pathway. Using immunofluorescence microscopy, the authors monitored ATG9 trafficking and distribution in conditions of serum starvation-induced autophagy combined with EGF stimulation. Under starvation conditions, wild-type ATG9 was dispersed in the cytosol; in response to EGF, ATG9 displayed partial localization at the plasma membrane followed by internalization along with EGFR, followed by a juxta-nuclear localization. In contrast, the SS1, SS2 and Y8F mutant displayed complete or partial resistance to EGF and remained largely at the plasma membrane.

Previous work showed that ATG9 traffics from the TGN to endosomes in a ULK1-dependent manner under stress conditions [11]. Accordingly, Zhou et al. decided to investigate the potential role of ULK1 in controlling the localization of ATG9. They identified a ULK1 phosphorylation site on ATG9 at Ser14. Phosphorylation at Ser14 was found to occur at a low level until cells were stressed, in contrast to Tyr8 that is highly phosphorylated in growing conditions. As with Tyr8, phosphorylation at Ser14 enhances the binding of ATG9 with the AP2 complex; Ser14 phosphorylation also promotes an ATG9-AP1 interaction. The authors found that an S14A mutant displayed a 
defect in starvation-dependent movement from the plasma membrane and TGN, leading to the conclusion that ULK1-dependent phosphorylation of ATG9 plays an important role in regulating the trafficking of this protein under autophagy-inducing conditions.

Having identified these two phosphorylation sites, Zhou et al. decided to examine whether they acted in a synergistic manner. Indeed, whereas phosphorylation of either site alone due to ectopic expression of SRC or ULK1 enhances the interaction between ATG9 and the AP1/2 complex, co-expression of these kinases results in an additive effect. Following internalization, ATG9 binds the RABGAP protein TBC1D5, and this interaction leads to autophagy induction [12]. Similar to the result seen with the AP1/2 complex, the ATG9TBC1D5 interaction was enhanced by ectopic expression of SRC and/or ULK1. Finally, Zhou et al. showed that phosphorylation of ATG9 at both the Tyr8 and Ser14 sites is required for maintaining proper autophagy under both basal conditions and in response to starvation-induced stress.

In summary, these findings reveal new connections between signals such as those involving the EGF receptor and SRC kinase and the regulation of autophagy, in particular through the ULK1 kinase. Although there are still many questions to be answered with regard to the role of ATG9 in membrane movement and phagophore expansion, these studies provide additional insight into the complex regulation needed to ensure the proper functioning of autophagy.

\section{Yuchen Feng ${ }^{1}$, Daniel J Klionsky ${ }^{1}$ \\ ${ }^{1}$ Life Sciences Institute and Department of Mo- lecular, Cellular and Developmental Biology, Uni- versity of Michigan, Ann Arbor, MI 48109, USA Correspondence: Daniel J Klionsky}

E-mail: klionsky@umich.edu

\section{References}

1 He C, Klionsky DJ. Annu Rev Genet 2009; 43:67-93.

2 Ge L, Melville D, Zhang M, et al. Elife 2013; 2:e00947.

3 Geng J, Nair U, Yasumura-Yorimitsu K, et al. Mol Biol Cell 2010; 21:2257-2269.

4 Hamasaki M, Furuta N, Matsuda A, et al. Nature 2013; 495:389-393.

5 Nair U, Jotwani A, Geng J, et al. Cell 2011; 146:290-302.

6 Ravikumar B, Moreau K, Jahreiss L, et al. Nat Cell Biol 2010; 12:747-757.

7 Yen WL, Shintani T, Nair U, et al. J Cell Biol 2010; 188:101-114.

8 Feng Y, Backues SK, Baba M, et al. Autophagy 2016; 12:648-658.

9 Papinski D, Schuschnig M, Reiter W, et al. Mol Cell 2014; 53:471-483.

10 Zhou C, Ma K, Gao R, et al. Cell Res 2017; 27:184-201.

11 Young ARJ, Chan EYW, Hu XW, et al. $J$ Cell Sci 2006; 119:3888-3900.

12 Popovic D, Dikic I. EMBO Rep 2014; 15:392-401. 